and linear algebra (2) provide theoretical computer scientists with adequate and powerful tools for future research.

\title{
REFERENCES
}

1. M. P. Schützenberger, Certain elementary families of automata, Proc. Sympos. on Math. Theory of Automata, Polytechnic Institute of Brooklyn, 1962, pp. 139-153.

2. __ Sur certain sous-monoïdes libres, Bull. Soc. Math. France 93 (1965), 209-222.

3. J. Berstel (Editor), Séries formelles en variables non-commutatives et applications, Publication of the L.I.T.P. University of Paris, May 1978.

Garard LALLEMENT

BULLETIN (New Series) OF THE

AMERICAN MATHEMATICAL SOCIETY

Volume, 1, Number 4, July 1979

(c) 1979 American Mathematical Society

0002-9904/79/0000-0315/\$01.75

Panorama des mathématiques pures. Le choix bourbachique, by Jean Dieudonné, Bordas, Dunod, Gauthier-Villars, Paris, France, 1977, xvii +302 pp., $150 \mathrm{FF}$.

What is mathematics? According to one often suggested definition, mathematics is what mathematicians do, and the answer, therefore, is a function of time. A more humble question is "what is mathematics now?", and even that is partly ambiguous. Mathematics comes in several packages, described by different labels. The subject of the book before us is not the large, family-sized package labelled "the mathematical sciences", which includes, for example, hydrodynamics, statistics, numerical analysis, and computer science. The subject is pure mathematics only; the stated purpose of the book is to give the student at the threshold of research a panoramic view of the pure mathematics that is alive today.

Another way of describing the subject is offered in the subtitle (and explained in more detail in the introduction): the book is about Bourbaki's choice. Bourbaki is a pseudonymous society of French mathematicians who for forty years have been publishing a systematic collection of expository texts, proceedings of seminars, and ex cathedra dicta. How did they decide which parts of mathematics merited attention and which ones did not? What principles have been guiding their choices? They have never answered the question in public, and the author (one of the charter members of the society) says that the only way to find the answer is to examine the results of the choices and to infer from the evidence at hand what must have motivated them. The author was for many years one of the chief Bourbaki scribes, but he insists that his conclusions are personal and do not represent the official Bourbaki point of view.

Be that as it may, what in fact is Bourbaki's choice? To answer the question, consider what every (almost every?) mathematical theory is like. It begins with a very special problem, or so the study of history teaches us, such for instance as the duplication of the cube. What happens next? Answer: there are several possibilities.

I. It could happen that efforts to solve the problem lead nowhere, and the theory is still-born; as examples consider the determination of the Fermat primes and the irrationality of Euler's constant. 
II. It could happen that the problem is solved, but leads to a dead end; most combinatory problems are like that.

III. It could happen that the techniques used in the solution serve to solve other problems, without, however, yielding a true understanding of the reason for their success. The result becomes known as a method; this is what happened to many problems in analytic number theory and the theory of finite groups.

IV. It could happen that a problem leads to a fecund general theory; typical current examples are Lie groups and algebraic topology.

V. It could happen that the theory to which the problem has led leads in turn to questions that are more and more special and isolated, so that the theory as a whole becomes emaciated; the theory of invariants, for instance, has passed through this stage several times.

VI. It could happen, finally, that a problem leads to an efficient axiomatization and thence to unmotivated investigations of modified axiom systems; in that case the theory becomes diluted beyond all interest and application (and, presumably, is doomed to death and disappearance). No examples are offered.

The author admits his complete objectivity in pointing out that most of the subjects expounded in the Bourbaki seminar belong to category IV, and, to a lesser extent, to category III. The subjects so chosen (here identified with pure mathematics) exhibit a remarkable unity. Hardly any idea in any theory fails to have repercussions in several others, and the traditional rigid division (algebra, geometry, and analysis) becomes inoperative.

The subjects are grouped into four levels (A, B, C, D) and arranged in order of their Bourbaki density, which is, for each subject, the ratio of the time and space allotted to the subject by Bourbaki to the entire mathematical literature about it. An importantly unimportant role is played by level D, which consists of the subjects of Bourbaki density zero. They are the theories that have remained essentially fixed for a long time, the ones that could be called classical. All the rest of the edifice is based on them; they are taught in the university; and they can be found in the already published parts of Bourbaki's Elements. The author admits that other people still do research in these subjects, and comments on the curious historical phenomenon of the division of a science into two parts that in practice mutually ignore each other, apparently without disturbing their separate developments in the least. The subjects are: set theory, general algebra, general topology, classical analysis, topological vector spaces, and integration.

Everything that $I$ have reported on so far appears in the book before page 1. There the business really begins: the panoramic view of all of pure mathematics is presented in twenty chapters (one per subject) varying in length from 6 pages (ergodic theory) to 34 (algebraic geometry). Some of the subjects are what you would expect (algebraic and differential topology, number theory, commutative aigebra), some have names that have not been universally adopted (analytic geometry, for example, means mainly the theory of functions of several complex variables and complex manifolds), and at least one surprised me by its presence (von Neumann algebras). Several subjects listed in Mathematical Reviews are absent. Examples: graphs, lat- 
tices, special functions, integral equations, calculus of variations, convexity.

Each chapter begins with a star graph; the subject of the chapter is in the center of the star, and other subjects are connected to the center by arrows pointing in or out (or both) according to the direction in which the influence has gone. The chapters get to work with no waste motion; there is almost no motivation and there are very few historical remarks. The style is merciless; it is more or less that of a one-paragraph abstract in the Notices of the American Mathematical Society (if you can imagine an abstract 285 pages long): bare bones definitions, name dropping, and sketchily (but carefully) described results follow each other clippety-clop. There is a long bibliography (199 items). In addition to the bibliography there is a short list of standard references that are used very frequently; they are the Bourbaki seminar, the Cartan seminar, invited addresses printed in this Bulletin, Symposia of the AMS, the Springer Lecture Notes series, and three recent International Congress proceedings (Stockholm 1962, Nice 1970, and Vancouver 1974-not Moscow 1966).

Every chapter ends with a paragraph entitled Relations with the natural sciences and a list of Initiators. The latter is a list of names (not references), divided into two sublists: the ones to whom the principal ideas of the subject are ascribed, and the others, the also-rans, who made substantial contributions to the theory.

The chapter on algebraic and differential topology is the first (A I). It starts with a description of the (homeomorphism) classification problem, and proceeds quickly through homotopy, CW complexes, cohomology, EilenbergMac Lane spaces, fiber spaces, characteristic classes, and spectral sequences, to manifolds (PL, etc.), the Poincaré conjecture, and cobordism. The relations with the natural sciences are described as indirect, mainly because the results of topology are qualitative existence and non-existence theorems and not explicit constructions. There is a long list of initiators stretching from Riemann to Papakyriakopoulos.

The chapter on abstract groups (B III) begins with generators and relations, goes through Chevalley groups and Tits systems, representation theory and characters, and the Brauer modular theory; it ends with a one-page report on finite simple groups. Relations with natural sciences: permutation groups and their representations intervene in several combinatorial questions that arise in applications. Initiators: from Cauchy to Janko.

The last chapter, for a last example, is on spectral theory (C III). It begins with the Jordan form of a matrix, and goes through the Riesz theory of compact operators, Banach algebras, $C^{*}$ algebras, the Gelfand transform, and Hilbert algebras, and ends with the spectral theory of normal operators on Hilbert space. Relations with the natural sciences: applications to physics, especially quantum theory. Initiators: from Sturm to Grothendieck.

It is impossible that a gigantic organizational report such as this could please everyone: sins of commission and omission, which may seem venial to some and mortal to others, are easy to find. Here is a tiny example: in the star graph of the von Neumann algebra chapter the subject is joined to ergodic theory with a double arrow (mutual influence), but in the ergodic chapter the graph indicates no connection with von Neumann algebras at all. 
As for omissions: von Neumann is not called an initiator of ergodic theory, neither Stark's name nor Szemeredi's is mentioned in the number theory chapter, G. A. Miller's name is not mentioned under abstract groups, Kos is not given any credit for ultraproducts, and Yosida's name doesn't appear anywhere. There is at least one other name that is conspicuous by its absence from all lists (initiators and others): J. Dieudonné.

Conclusion. Dieudonné has given us an unusual book, a remarkable book, a very personal book, an argumentative book, in many ways a dogmatic book, a controversial book, an informative book, and without doubt an interesting book.

P. R. HALMOS

\section{ERRATA, Volume 84}

On pages 1351 and 1360 of Volume 84 of the Bulletin of the American Mathematical Society, November, 1978, the publisher of the books "The theory of information and coding: $\boldsymbol{A}$ mathematical framework for communication" and "Catastrophe theory: Selected papers, 1972-1977," was incorrectly listed. It should have read "Addison-Wesley, Reading, Mass." 